\title{
Compact Planar Ultra-Wide Pass-Band Filters With Source-Load Coupling and Impedance Stubs
}

\author{
Marjan Mokhtaari ${ }^{1}$, Jens Bornemann ${ }^{1}$ and Smain Amari ${ }^{2}$ \\ ${ }^{1}$ University of Victoria, Victoria, BC, Canada V8W 3P6 \\ ${ }^{2}$ Royal Military College of Canada, Kingston, ON, Canada K7K 7B4
}

\begin{abstract}
New configurations of folded and compact planar ultra-wideband filters with sourceload cross coupling and impedance stubs are presented. Stepped impedance resonators and coupled-line sections as inverter circuits form the basic filter structures. High-low impedance lines connect individual resonators. Effective techniques such as open-stub lines and source-load cross coupling are utilized in order to add return-loss poles in the pass-band and create transmission zeros in the lower/upper stop-band region. Several filters are designed with $3 \mathrm{~dB}$ fractional bandwidths of 80 percent, computed insertion losses better than $1 \mathrm{~dB}$ and return losses larger than $10 \mathrm{~dB}$. Rejection levels in the upper/lower stop-bands are better than $35 \mathrm{~dB}$. The designs are verified by results obtained with commercial electromagnetic software packages.
\end{abstract}

Index Terms - Filter design, ultra-wideband filters, stepped impedance resonators, microstrip filters.

\section{INTRODUCTION}

Ultra Wide-Band (UWB) technology has received increased attention since the release of the 3.1-10.6 GHz frequency band. Especially for wireless communications applications, ultra-wide pass-band filters are in demand. Their specifications include high-performance electrical responses but, at the same time, they must be mass-producible at low cost and small size.

Parallel-coupled transmission lines for ultrabroadband microstrip pass-band filters with bandwidths up to 70 percent are presented in [1], [2]. However, the filter size is not compact, and the design lacks transmission zeros to increase selectivity. A similar technique is used in [3], [4] and includes impedance steps and coupled-line sections as inverter circuits. The bandwidths of these structures are around 50 and 110 percent, respectively. A similar version in coplanarwaveguide technology [5] achieves 104 percent bandwidth. While these latest results are excellent, the number of possible transmission zeros, as required for high-selectivity filters, is very limited - as is the stopband attenuation towards higher frequencies.

Ring resonators with additional stubs are introduced in [6]. Control of the attenuation pole frequency is achieved by adjusting both the ring and stub impedances. In order to increase the bandwidth and number of transmission zeros, multi-stage resonator arrangements are required, which increases component size. Dual-mode stopband ring resonators with two tuning stubs are used in [7] to construct a wide pass-band with two sharp stop-bands. Due to the characteristics of the band-stop resonators, however, this filter has additional pass-bands to the left and right of the stop-bands.

A compact ultra-wideband LTCC filter employing four resonators and 1-to-4 cross coupling is introduced in [8]. However, the bandwidth is only 50 percent. Other ultrawideband filters with similar characteristics and smaller bandwidths have been designed in coplanar waveguide [9], [10], substrate integrated waveguide [11] and microstrip technology [12]. A new ultra-wide band filter structure with more than 100 percent bandwidth and wide stop-band regions is introduced in [13]. It uses cascaded broadside-coupled microstrip-coplanar waveguide structures but requires an additional low-pass filter to suppress the spurious pass-band in the upper band region.

This paper focuses on modified approaches of the impedance-step, additional-stub and parallelcoupled microstrip-line concept for the design of very compact broadband filters. Cross coupling is introduced to generate additional transmission zeros. Wide out-of-band rejection is obtained by controlling the number and positions of transmission zeros close to the lower and upper skirt of the passband. Ultra-wideband filter structures with improved filter characteristics such as sharp selectivity and enhanced bandwidth are presented.

\section{THEORY AND DESIGN}

Let us first consider in Fig. 1a the layout of an ultra-wideband filter (similar to the one in [4]) including the centered transmission-line (TL) resonator with impedance steps and two sections of parallel-coupled microstrip lines (PCML's), 
which act as admittance inverters (J). Fig. 1b depicts the equivalent circuit topology.

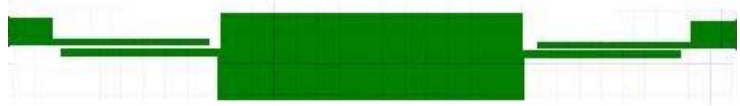

(a)

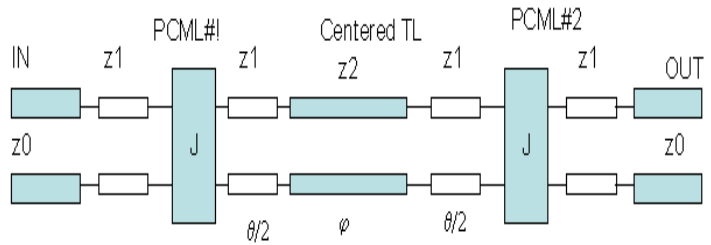

(b)

Fig. 1. Layout (a) of ultra-wideband filter (similar to [4]) and equivalent circuit topology (b).

Using transmission line theory, and under the simplified assumption that the impedance of the centered TL equals that of the input/output lines, the reflection coefficient can be formulated as a closed-form function

$$
S_{11}=\frac{j\left(1-\bar{J}^{4}\right) \cdot \tan (\Phi)}{2 \bar{J}^{2}+j\left(1+\bar{J}^{4}\right) \cdot \tan (\Phi)}
$$

where $\bar{J}=J / Y_{0}$ is the normalized inverter and the total electrical length is

$$
\Phi=\frac{\theta}{2}+\phi+\frac{\theta}{2}
$$

For a broadband filter design, we use the fundamental and the next two harmonic resonances of the stepped impedance resonator. The two PCML's create two more in-band reflection zeros. Thus a broadband five-pole bandpass filter is created with a single line resonator.

The lengths of the PCML sections are a quarterwavelength at center frequency with very tight coupling to create a broad pass-band. The centered transmission line is half a wavelength long with low characteristic impedance. After obtaining initial dimensions for given substrate height and permittivity, the design proceeds by adjusting the characteristic impedances and gaps in the PCML's - towards tight coupling - and the characteristic impedance of the middle transmission line towards a low impedance profile. The resonant length is adjusted to compensate for the phase deviations introduced by the inverters.

In order to increase the bandwidth of this filter configuration, a new high impedance resonator is added according to Fig. 2. The additional resonator increases the bandwidth by up to ten percent and, at the same time, bypasses the tripleresonance stepped impedance resonator to generate up to three transmission zeros above the passband. The location of these transmission zeros can be used to improve the upper skirt of the filter and/or the stop-band towards higher frequencies. The length and impedance characteristic of the new resonator may be defined such that it is tightly coupled and adds a reflection zero in the lower pass-band.

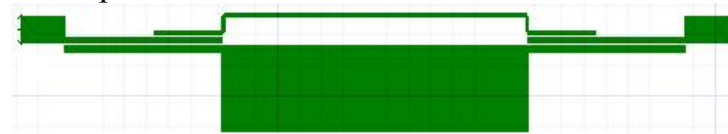

Fig. 2. Modified ultra-wideband filter configuration with cross coupling.

In order to obtain a more compact filter design, the structure in Fig. 1a can be folded. Moreover, parallel open-ended stubs can be added to the centered transmission line. This is shown in Fig. 3. The stub creates a strong reflection zero at the upper edge of the passband and a sharp transmission zero in the upper rejection band. It also attenuates the second harmonic of the passband and broadens the stop-band region up to the third passband harmonic. The stubs should have resonance lengths close to a quarter-wavelength at the upper passband frequency as well as high impedances to reduce their impact on the passband.

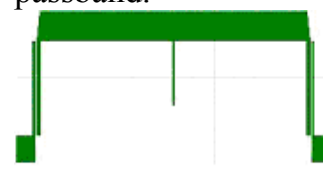

(a)

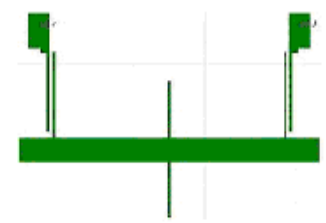

(b)
Fig. 3. Folded ultra-wideband filter configurations with a single stub (a) and dual stubs (b).

Moreover, to place transmission zeros in the lower/upper rejection band, a direct source-load cross coupling can be applied to the folded structures. This is shown in Fig. 4a and includes an additional stub in Fig. 4b. Four extra transmission zeros, two on each side of the pass band, are created and controlled by the sourceload coupling coefficient.

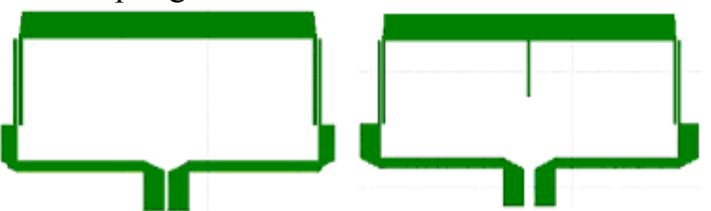

(a)

(b)

Fig. 4. Folded ultra-wideband filters with source-load coupling (a) and added single stub (b).

\section{RESULTS}

The design guidelines presented in the previous section are applied to the various filter configurations introduced in Figs. 2-4. All filters are designed on RT6006 substrate with a height of $635 \mu \mathrm{m}(25 \mathrm{mil})$ and are verified by the two commercial field solvers Ansoft Designer ${ }^{\odot}$ and IE3D ${ }^{\odot}$. Note that we refrained from using any 
aperture compensation in the ground plane of the circuits, which eases the fabrication process.

Fig. 5 depicts the results obtained with the ultrawideband filter configuration in Fig. 2. As predicted, up to three transmission zeros can be placed in the upper rejection band (Fig. 5b) by controlling the length of the new resonator and the source-load coupling (as compared to Fig. 5a). This creates a wide stop-band and a sharp skirt on the upper side of the pass-band. The return loss is more than $12 \mathrm{~dB}$ from 3.2 to $7 \mathrm{GHz}$. The $3 \mathrm{~dB}$ bandwidth is around 80 percent $(3-7.1 \mathrm{GHz})$, and the predicted pass-band insertion loss is less than $1 \mathrm{~dB}$. The small peak at around $3.5 \mathrm{GHz}$ is believed to be caused by a weak coupling between the center section of the stepped impedance resonator and the bridging resonator (c.f. insets in Fig. 5).

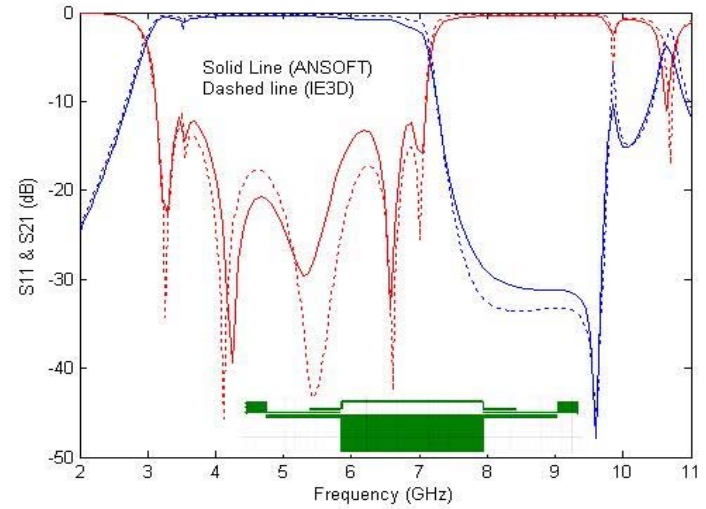

(a)

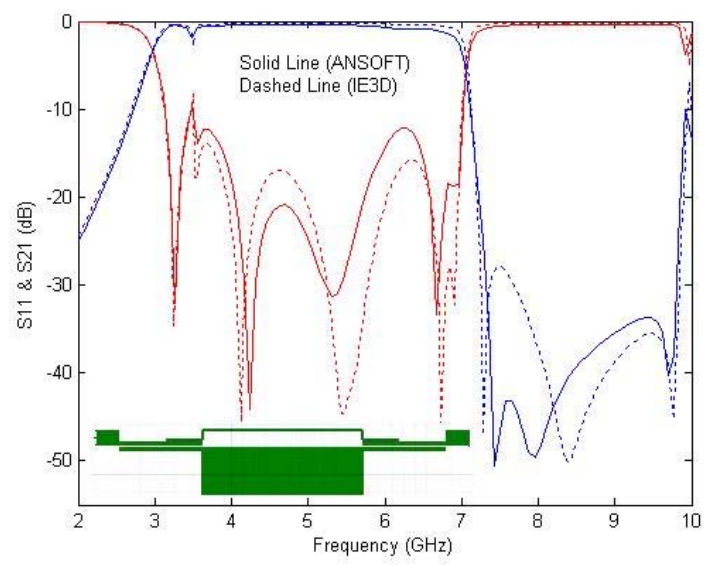

(b)

Fig. 5. Performances of the modified ultra-wideband filter configuration according to Fig. 2 with a short additional resonator and weak cross coupling (a) and longer resonator and strong cross coupling (b).

The results of configurations with additional stubs (Fig. 3) are shown in Fig. 6. Apparently, the additional stubs increase the bandwidth for a single resonator up to eight percent and insert a strong transmission zero at the upper edge of the pass-band. It also has a large attenuation impact on the second harmonic of the passband. In Fig. $6 \mathrm{a}$, the reflection coefficient is better than $-13 \mathrm{~dB}$ from 4.2 to $9.5 \mathrm{GHz}$ with a predicted insertion loss less than $0.65 \mathrm{~dB}$. The corresponding values in Fig. $6 \mathrm{~b}$ are: $15 \mathrm{~dB}$ from 3.2 to $7.3 \mathrm{GHz}$, and less than $0.5 \mathrm{~dB}$. The configuration in Fig. $6 \mathrm{~b}$ has a compact size due to the folded structure and better upper stop-band rejection up to the third passband harmonic.

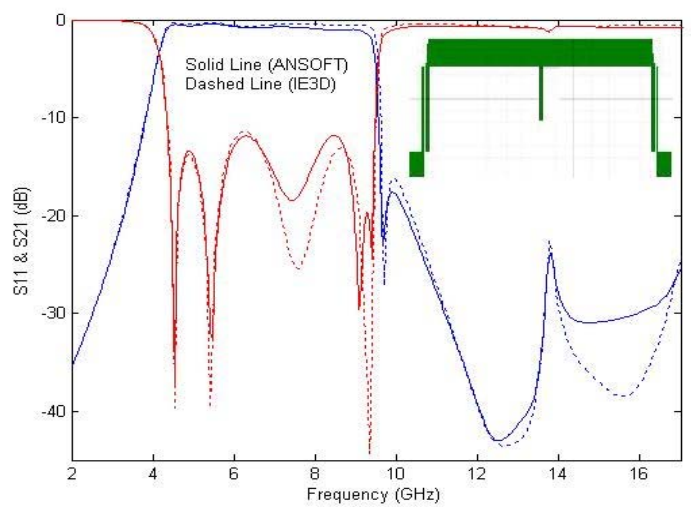

(a)

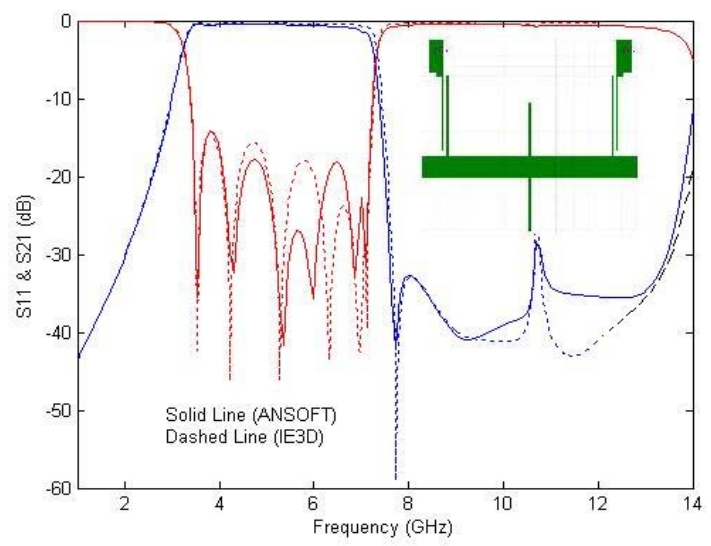

(b)

Fig. 6. Responses of ultra-wideband filter configurations according to Fig. 3; bandwidth of 4.2 9.5GHz (a), $3.2-7.3 \mathrm{GHz}$ (b).

Direct source-load coupling can be applied to the ultra-wideband filter structure to create transmission zeros on both sides of the pass-band. Fig. 7 shows the performance of two UWB filters according to Fig. 4 with and without the additional stub. Two extra zeros are located on each side of the passband due to the cross coupling; their locations can be adjusted by the source-load coupling coefficient (length). Fig. $7 \mathrm{~b}$ also shows one of the additional transmission zeros generated by the stub. The $3 \mathrm{~dB}$ bandwidth is from 3 to 7.1 $\mathrm{GHz}$ with a return loss of $11 \mathrm{~dB}$.

\section{CONCLUSION}

Guidelines for the design of compact planar ultra-wide pass-band filters are presented. The 
core section is based on three resonances of a stepped impedance resonator and two tightly coupled microstrip lines. Adding an additional cross-coupling resonator or folding the filter to add source-load coupling presents viable options for increasing the bandwidth and improving the selectivity. Additional impedance stubs can be used to create transmission zeros close to the upper band edge and in the upper stopband. Thus ultra-broad pass-band filters with transmission zeros on both sides of the passband can be realized by using a single stepped impedance resonator. The designs refrain from using groundplane apertures and, therefore, do not complicate fabrication. All performances are verified by two different EM packages.

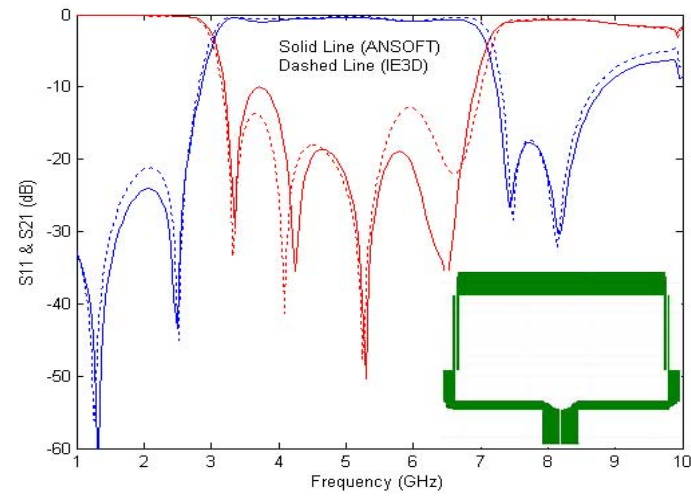

(a)

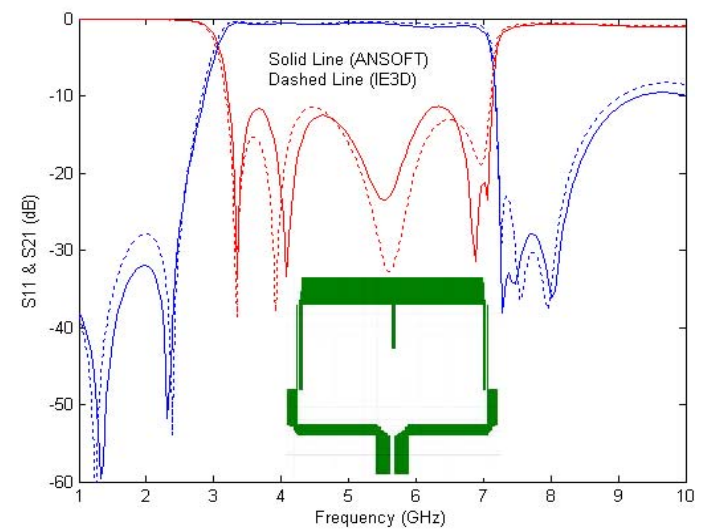

(b)

Fig. 7. Performances of ultra-wideband filter configurations with direct source-load coupling according to Fig. 4a (a) and Fig. 4b (b).

\section{REFERENCES}

[1] L. Zhu, H. Bu and K. Wu, "Aperture compensation technique for innovative design of ultra-broadband microstrip bandpass filter", 2000 IEEE MTT-S Int. Microwave Symp. Dig., pp. 315-318, June 2000.

[2] L. Zhu, H. Bu and K. Wu, "Aperture compensation and multipole generation techniques leading to the emergence of a new planar filter", Proc. 2000 Asia-Pacific Microwave Conf., pp. 1310-1314, Dec. 2000.

[3] W. Menzel, L. Zhu, K. Wu and F. Bögelsack, "On the design of novel compact broad-band planar filters", IEEE Trans. Microwave Theory Tech., Vol. 51, pp. 364-370, Feb. 2003.

[4] L. Zhu, S. Sun and W. Menzel, "Ultra-wideband (UWB) bandpass filters using multiple-mode resonator", IEEE Microwave Wireless Comp. Lett., VOl. 11, pp. 796-798, Nov. 2005.

[5] J.Gao, L.Zhu, W. Menzel and F. Bögelsack, "Short-circuited CPW multiple-mode resonator for ultra-wideband (UWB) bandpass filter", IEEE Microwave Wireless Comp. Lett., Vol. 16, pp. 104-106, Mar. 2006.

[6] H. Ishida and K.Araki, "Design and analysis of UWB band pass filter with ring filter", 2004 IEEE MTT-S Int. Microwave Symp. Dig., pp. 13071310, June 2004.

[7] L.-H. Hsieh and K. Chang, "Compact, low insertion-loss, sharp-rejection, and wide-band microstrip bandpass filters", IEEE Trans. Microwave Theory Tech., Vol. 51, pp. 1241-1246, Apr. 2003.

[8] C.-W. Tang, C.-C. Tseng, H.-H. Liang and S.-F. You, "Development of ultra-wideband LTCC filter", Proc. 2005 IEEE Int. Conf. UltraWideband, pp. 320-322, Sept. 2005.

[9] B.-S. Kwon, S.-S. Myoung, Y.-H. Kim and J.-G. Yook, "Co-planar waveguide filter with ground perforation for ultra-wideband system", Proc. 2005 Asia-Pacific Microwave Conf., 4 pp., Dec. 2005.

[10] Y.-K. Kuo, C.-H. Wang and C.H. Chen, "Novel reduced-size coplanar-waveguide bandpass filters", IEEE Microwave Wireless Comp. Lett., Vol. 11, pp. 65-67, Feb. 2001.

[11] Z.C. Hao, W. Hong, H. Li, H. Zhang and K. Wu, "A broadband substrate integrated waveguide (SIW) filter", 2005 IEEE AP-S Int. Symp. Dig., pp. 598-601, July 2005.

[12] C. Nguyen, "Development of new miniaturised bandpass filter having ultrawide bandwidth", IEE Electronics Lett., Vol. 30, pp. 767-768, May 1994.

[13] K. Li, "UWB bandpass filter: Structure, performance and application to UWB pulse generation", Proc. 2005 Asia-Pacific Microwave Conf., pp. 79-82, Dec. 2005. 